\title{
TIME-DEPENDENT WKB EXPANSION FOR THE NON-RELATIVISTIC COULOMB PROPAGATOR
}

\author{
S.M. BLINDER \\ Department of Chemistry, University of Michigan, Ann Arbor, MI 48109, USA
}

Received 1 October 1986; in final form 5 December 1986

\begin{abstract}
An approximation to the non-relativistic Coulomb propagator, correct to second order in $\hbar$, is derived. The result is expressed in terms of the auxiliary variables introduced in the solution of the corresponding Hamilton-Jacobi equation. Higher-order contributions can be computed as required, but no explicit summation of the expansion has yet been found.
\end{abstract}

In a recent article [1] we described a time-dependent WKB expansion technique for propagators which do not conform to the simple Feynman structure $K$ $=F(t) \exp (\mathrm{i} S / \hbar)$. The method was applied to the radial propagators for multidimensional harmonic oscillators, yielding explicit summations both as asymptotic expansions in ascending powers of $\hbar$ and as convergent expansions in descending powers of $\hbar$. These sums were, in fact, identified as alternative representations for a Bessel function.

In the present paper we shall apply this method to the non-relativistic Coulomb propagator, for which no closed form yet exists (for a review of the current status of the problem, see ref. [2]). This remains a longmissing element in Feynman's path-integral formulation of quantum mechanics. Further study of the Coulomb propagator, representing the prototype system in the quantum theory of matter, is intrinsically valuable. In addition, the potential for application to atomic physics and quantum chemistry is yet to be fully exploited. In earlier work we have solved for the zeroth-order (classical) [3] and first-order (semiclassical) approximations [4]. We will arrive at an expansion in ascending powers of $\hbar$ which we will exhibit explicitly to second order. We are not able at this time to sum the series.

The Coulomb propagator is the solution of the time-dependent Schrödinger equation

$\mathrm{i} \partial K / \partial t+\frac{1}{2} \nabla_{1}^{2} K+\left(Z / r_{1}\right) K=0$ subject to the initial condition

$K\left(r_{1}, r_{2}, 0\right)=\delta\left(r_{1}-r_{2}\right)$.

We employ atomic units, $\hbar=e=m=1$, but restore $\hbar$ for use as an expansion parameter. It has been shown [2] that the propagator can be reduced to the form

$K(x, y, t)=-\frac{1}{\pi(x-y)}\left(\frac{\partial}{\partial x}-\frac{\partial}{\partial y}\right) k(x, y, t)$

in terms of the variables

$x=r_{1}+r_{2}+r_{12}, y=r_{1}+r_{2}-r_{12}$,

such that (1) reduces to a pseudo one-dimensional form

$\frac{1}{2} \mathrm{i} k_{t}+k_{x x}+(Z / x) k=0$

subject to the initial condition

$k(x, y, 0)=\delta(x-y)$.

The variables $x$ and $y$ were introduced by Hostler, who first derived a closed form for the (time-independent) non-relativistic Coulomb Green's function [5]. The dependence on just these two combinations of variables, whereas rotational symmetry alone would imply three variables, say $r_{1}, r_{2}, r_{12}$, is a consequence of the $\mathrm{SO}(4)$ [or $\mathrm{SO}(3,1)$ ] dynamical symmetry of the Coulomb problem. This is connected as well with the additional constant of the mution: the RungeLenz vector [6].

As shown by Feynman and others [7], for Hamil- 
tonians expressible as quadratic forms in generalized coordinates and momenta, the propagator has the structure

$K\left(q_{1}, q_{2}, t\right)=F(t) \exp \left[\mathrm{i} S\left(q_{1}, q_{2}, t\right) / \hbar\right]$

in which $S$ is the classical action, the solution of the corresponding Hamilton-Jacobi equation. The modulating function $F$, dependent on $t$ alone, usually follows easily from the time-dependent Schrödinger equation with its associated initial condition.

For non-harmonic potentials, including the Coulomb problem, the simple structure (7) no longer suffices. We propose to represent the propagator $k(x, y, t)$ (cf. eq. (3)) in the slightly more general form

$k(x, y, t)=f(x, y, t) \exp [\mathrm{i} S(x, y, t) / \hbar]$,

with the preexponential factor now free to contain dependence on $x$ and $y$, as well as $t$. Substituting (8) into (5) gives

$$
\begin{aligned}
& \left(\frac{1}{2} S_{t}+S_{x}^{2}-Z / x\right) f+\mathrm{i} \hbar\left(\frac{1}{2} f_{\mathrm{t}}+2 S_{x} f_{x}+S_{x x} f\right) \\
& +\hbar^{2} f_{x x}=0 .
\end{aligned}
$$

In the limit $\hbar \rightarrow 0$, eq. (9) reduces to the classical Hamilton-Jacobi equation for the Kepler-Coulomb problem,

$\frac{1}{2} S_{t}+S_{x}^{2}-Z / x=0$.

The solution, which we obtained a long time ago [3], can be expressed as

$S=\nu[\sinh (\lambda-\mu) \cosh (\lambda+\mu)+3(\lambda-\mu)]$,

in terms of the auxiliary variables $\lambda, \mu, \nu$ defined by the following network of implicit relations:

$Z x=4 \nu^{2} \sinh ^{2} \lambda, \quad Z y=4 \nu^{2} \sinh ^{2} \mu$,

$Z^{2} t=2 \nu^{3}[\sinh (\lambda-\mu) \cosh (\lambda+\mu)-(\lambda-\mu)]$.
As done in ref. [1], we assume an expansion of $f(x, y, t)$ in ascending powers of $\hbar$ :

$f(x, y, t)=\sum_{n=0}^{\infty}(\mathrm{i} \hbar)^{n} f^{(n)}(x, y, t)$.

With use of (10) and (13), eq. (9) is transformed to a recursive relation for the $f^{(n)}(x, y, t)$;

$\frac{1}{2} f_{t}^{(n)}+2 S_{x} f_{x}^{(n)}+S_{x x} f^{(n)}=f_{x x}^{(n-1)}$

For further progress, we must reexpress eq. (14) in terms of the $\lambda, \mu, \nu$ variables. To transform the derivatives we require the elements of the Jacobian matrix $\partial(\lambda, \mu, \nu) / \partial(x, y, t)$ enumerated in the array given in table 1. For compactness, the following abbreviations will be employed: $S_{\lambda}=\sinh \lambda, C_{\lambda}=\cosh \lambda, S_{\mu}$ $=\sinh \mu, C_{\mu}=\cosh \mu$. Also we have defined

$j(\lambda) \equiv S_{\lambda}^{3}+3 S_{\lambda}-3 \lambda C_{\lambda}$,

$J(\lambda, \mu) \equiv C_{\mu} j(\lambda)-C_{\lambda} j(\mu)$.

The following identities are readily verified:

$C_{\lambda} j^{\prime}(\lambda)=S_{\lambda} j(\lambda)+2 S_{\lambda}^{4}$

$C_{\lambda} J_{\lambda}=S_{\lambda} J+2 S_{\lambda}^{4} C_{\mu} \quad\left(J_{\lambda} \equiv \partial J / \partial \lambda\right)$,

$C_{\mu} J_{\mu}=S_{\mu} J-2 S_{\mu}^{4} C_{\lambda} \quad\left(J_{\mu} \equiv \partial J / \partial \mu\right)$.

The $x$-derivatives of the action function can now be expressed as

$S_{x}=\frac{C_{\lambda}}{2 \nu S_{\lambda}}, \quad S_{x x}=\frac{1}{16 \nu^{3} S_{\lambda}^{2}}\left(\frac{J_{\lambda}}{J}-\frac{C_{\lambda}}{S_{\lambda}}\right)$.

Also

$\frac{1}{2} \frac{\partial}{\partial t}+2 S_{x} \frac{\partial}{\partial x}=\frac{1}{8 \nu^{3} S_{\lambda}^{2}} \frac{\partial}{\partial \lambda}$.

Remarkably, eq. (14) with $n=0$ reduces to an ordinary differential equation for $f^{(0)}$,

Table 1 
$\frac{1}{8 \nu^{3} S_{\lambda}^{2}}\left[\frac{\partial f^{(0)}}{\partial \lambda}+\frac{1}{2}\left(\frac{J_{\lambda}}{J}-\frac{C_{\lambda}}{S_{\lambda}}\right) f^{(0)}\right]=0$.

The solution is of the form

$f^{(0)}=\left(S_{\lambda} / J\right)^{1 / 2} \times$ function of $\mu, \nu$.

The symmetry between $\lambda$ and $\mu$ implies further that

$f^{(0)}=\left(S_{\lambda} S_{\mu} / J\right)^{1 / 2} \times$ function of $\nu$.

It seems reasonable, given the structure of the above formulas involving the auxiliary variables, that the dependence on $\nu$ takes the form; const. $\nu^{\alpha}$. In fact, for $\alpha=-3 / 2$ and

$f^{(0)}=\left(Z^{2} / 4 \pi \mathrm{i}\right)^{1 / 2} \nu^{-3 / 2}\left(S_{\lambda} S_{\mu} / J\right)^{1 / 2}$,

the semiclassical approximation to the Coulomb propagator

$k \approx f^{(0)} \exp (\mathrm{i} S / \hbar)$

satisfies the initial condition (6). It is not necessarily true, however, that the same value of $\alpha$ is appropriate for the complete $\hbar$ expansion, which is an asymptotic series.

With use of (19), eq. (14) can be recast in the form

$f^{(0)} \frac{\partial}{\partial \lambda}\left(\frac{f^{(n)}}{f^{(0)}}\right)=8 \nu^{3} S_{\lambda}^{2} f_{x x}^{(n-1)}$.

The $x$-derivatives in (24) must now be expressed in terms of $\lambda, \mu, \nu$. For functions of the form

$f=\nu^{\alpha} \phi(\lambda, \mu)$

we find from the preceding transformation formulas

$$
\begin{aligned}
\frac{\partial f}{\partial x} & =\nu^{\alpha-2}\left[\left(\frac{1}{8 S_{\lambda} C_{\lambda}}+\frac{S_{\lambda}^{2} C_{\mu}}{4 C_{\lambda} J}\right) \phi_{\lambda}\right. \\
& \left.+\frac{S_{\lambda} S_{\mu}}{4 J} \phi_{\mu}-\alpha \frac{S_{\lambda} C_{\mu}}{4 J} \phi\right]
\end{aligned}
$$

The requisite derivatives and integrals of the products $\nu^{\alpha} \dot{S}_{\lambda}^{A} C_{\lambda}^{B} S_{\mu}^{C} C_{\mu}^{D} J^{N}$

are evaluated in symbolic form by a computer program. Thereby eq. (24) can be solved sequentially to any order $n$. We display explicitly only the function, $f^{(1)}$, which has the structure

$f^{(1)}=\nu^{-1} f^{(0)} \sum_{m=0}^{3} \phi^{(1, m)} / J^{m}$, with

$$
\begin{aligned}
\phi^{(1,3)} & =-\frac{5}{12} \frac{\left(S_{\lambda}^{5} C_{\mu}^{3}-C_{\lambda}^{3} S_{\mu}^{5}\right)^{2}}{C_{\lambda}^{3} C_{\mu}^{3}}, \\
\phi^{(1,2)} & =\frac{5}{8} \frac{\left(S_{\lambda}^{5} C_{\mu}^{5}-C_{\lambda}^{5} S_{\mu}^{5}\right)}{C_{\lambda}^{3} C_{\mu}^{3}}+\frac{1}{3} \frac{\left(S_{\lambda}^{5} C_{\mu}^{3}-C_{\lambda}^{3} S_{\mu}^{5}\right)}{C_{\lambda} C_{\mu}} \\
+\frac{1}{4} & \frac{\left(S_{\lambda}^{5} C_{\mu}-C_{\lambda} S_{\mu}^{5}\right)}{C_{\lambda} C_{\mu}}, \\
\phi^{(1,1)} & =-\frac{5}{16} \frac{\left(S_{\lambda}^{4} C_{\mu}^{4}+C_{\lambda}^{4} S_{\mu}^{4}\right)}{C_{\lambda}^{3} C_{\mu}^{3}}+\frac{5}{8} \frac{\left(S_{\lambda}^{2} C_{\mu}^{2}+C_{\lambda}^{2} S_{\mu}^{2}\right)}{C_{\lambda} C_{\mu}} \\
-\frac{1}{8} & \frac{S_{\lambda}^{2} S_{\mu}^{2}}{C_{\lambda} C_{\mu}}-\frac{1}{24} C_{\lambda} C_{\mu}, \\
\phi^{(1,0)} & =\frac{1}{16} \frac{\left(S_{\lambda} C_{\mu}-C_{\lambda} S_{\mu}\right)}{S_{\lambda} S_{\mu}}-\frac{9}{32} \frac{\left(S_{\lambda} C_{\mu}-C_{\lambda} S_{\mu}\right)}{C_{\lambda} C_{\mu}} \\
+\frac{1}{48} & \frac{\left(S_{\lambda}^{3} C_{\mu}^{3}-C_{\lambda}^{3} S_{\mu}^{3}\right)}{C_{\lambda}^{3} C_{\mu}^{3}} .
\end{aligned}
$$

Each increment of $n$ generates four additional negative powers of $J(\lambda, \mu)$. The successive orders $f^{(n)}$ increase rapidly in complexity and follows no readily discernible pattern.

\section{References}

[1] S.M. Blinder, Chem. Phys. Letters 123 (1986) 47.

[2] S.M. Blinder, Intern. J. Quantum Chem. Symp. 18 (1984) 293.

[3] S.M. Blinder, J. Math. Phys. 16 (1975) 2000.

[4] S.M. Blinder, Phys. Rev. Letters 52 (1984) 1771.

[5] L. Hostler and R.H. Pratt, Phys. Rev. Letters 10 (1963) 469;

L. Hostler, J. Math. Phys. 5 (1964) 591.

[6] L. Hostler, J. Math. Phys. 8 (1967) 642.

[7] R.P. Feynman, Rev. Mod. Phys. 20 (1948) 367; R.P. Feynman and A.R. Hibbs, Quantum mechanics and path integrals (MoGraw-Hill, New York, 1965); S. Albeverio and R. H申egh-Krohn, Mathematical theory of Feynman path integrals, in: Lecture notes in mathematics, Vol. 523 (Springer, Berlin, 1976). 